\section{RMD Open}

Rheumatic \&

Musculoskeletal Diseases

\title{
Cancer immunotherapy-induced rheumatic diseases emerge as new clinical entities
}

\author{
Laura C Cappelli, Ami A Shah, Clifton O Bingham III
}

To cite: Cappelli LC, Shah AA, Bingham CO. Cancer immunotherapyinduced rheumatic diseases emerge as new clinical entities. RMD Open 2016;2: e000321. doi:10.1136/ rmdopen-2016-000321

- Prepublication history for this paper is available online. To view these files please visit the journal online (http://dx.doi.org/10.1136/ rmdopen-2016-000321).

Received 4 August 2016 Revised 29 August 2016 Accepted 7 September 2016

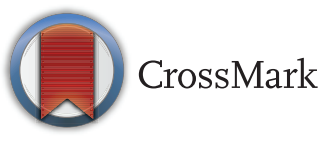

Department of Medicine, Division of Rheumatology, Johns Hopkins University, Baltimore, Maryland, USA

Correspondence to Dr Clifton 0 Bingham III; cbingha2@jhmi.edu

\section{ABSTRACT}

Immune checkpoint inhibitors (ICls) are newly approved treatments for advanced malignancies that are increasing survival. The mechanism of these drugs, non-specifically activating $T$ cells, also leads to immune-mediated damage of tissue or immune-related adverse events (IRAE). IRAEs with rheumatic phenotypes are increasingly being recognised. Inflammatory arthritis, sicca syndrome, inflammatory myopathy, vasculitis and lupus nephritis have been described as a result of ICls. Use of ICls will be expanding in the coming years for several reasons. ICls will be used in earlier stage cancer, for more indications, and additional drugs will be approved. The rheumatologist plays a critical role in evaluating and treating these patients. The expertise of rheumatologists in evaluating rheumatic signs and symptoms and treating patients with immunosuppression are critical in ensuring the optimal outcomes for patients with rheumatic IRAE. Collaboration between oncology and rheumatology for clinical care and research will enhance understanding of these new disease entities.

\section{INTRODUCTION}

Cancer therapy has evolved dramatically in recent years with the approval of immune checkpoint inhibitors (ICIs) as immunotherapies to treat advanced stage disease. ${ }^{1}$ These drugs work by inhibiting negative regulation of T cells, thus leading to heightened antitumour responses. Ipilimumab, targeting CTLA-4, nivolumab and pembrolizumab, targeting PD-1, and atezolizumab, targeting PD-L1, are FDA-approved, with the first three drugs also approved in the European Union. ICIs have improved survival for several cancers and, in a subset of patients, can lead to lasting tumour regression or complete remission. Currently approved indications include metastatic melanoma, non-small cell lung cancer (NSCLC), renal cell carcinoma (RCC), Hodgkin's lymphoma and urothelial

\section{Key messages}

What is already known about this subject?

- Immune related adverse events from immune checkpoint inhibitors used to treat cancer can resemble classic rheumatic diseases.

How might this impact on clinical practice?

- Rheumatologists have an important role to play in the clinical care and research of immune related adverse events.

carcinoma, ${ }^{2-7}$ with many other malignancies being studied and across all tumour stages.

Though these drugs have improved outcomes for some advanced malignancies, they are not without consequences. Owing to their non-specific mechanism of activating $\mathrm{T}$ cells, the main toxicities of ICIs are due to immunologically mediated and inflammatory damage of tissues, collectively referred to as immune-related adverse events (IRAEs). ${ }^{8}$ IRAEs have been described that affect nearly every organ system. Colitis, various rashes, pneumonitis, hepatitis, encephalopathy, neuropathy, thyroiditis and hypophysitis are some of the wide-ranging adverse effects attributed to ICIs. Biopsies from colitis have demonstrated diverse inflammatory cell infiltrates, ${ }^{9}$ and development of colitis has been shown to be associated with elevated levels of IL-17, ${ }^{10}$ highlighting the relationship between immune activation and tissue damage. In the 5 years since the first ICI was approved, reports have described a variety of IRAEs with clinical manifestations similar to rheumatic disease. Inflammatory arthritis has been described in case reports ${ }^{11}{ }^{12}$ and a case series. ${ }^{13}$ In our own experience evaluating nine patients treated with ICIs who developed inflammatory arthritis, we observed three major types: polyarticular arthritis involving small and large joints similar to rheumatoid arthritis, a reactive arthritis-like 
syndrome with urethritis, conjunctivitis and oligoarthritis, and a large joint predominant seronegative spondyloarthritis. ${ }^{13}$ Many of these patients had severe, highly inflammatory disease requiring higher doses of corticosteroids to control than traditional forms of inflammatory arthritis. Several continued to have symptoms of inflammatory arthritis months to over a year after immunotherapy was withdrawn. Sicca syndrome was also reported in the same case series with patients experiencing severe salivary hypofunction. Additional rheumatic manifestations of ICIs include vasculitis, ${ }^{14}{ }^{15}$ inflammatory myopathy, ${ }^{16}{ }^{17}$ eosinophilic fasciitis ${ }^{18}$ and lupus nephritis. ${ }^{19}$ Within vasculitis, giant cell arteritis and single organ vasculitis have been described. Cases of inflammatory myopathy similar to dermatomyositis and polymyositis are both reported. There may be other rheumatic IRAEs resulting from treatment of ICIs that have not yet been described in the published literature.

\section{AN UNDER-REPORTED PROBLEM}

All the aforementioned rheumatic IRAEs have only been described in case reports or small series. Many clinical trials that have published toxicity data either do not report rheumatic IRAE, do not provide clinical descriptions of rheumatic IRAE, or only report high-grade adverse events, thus potentially excluding events such as inflammatory arthritis. Additionally, no prospective cohort study has evaluated a population of patients treated with ICIs for the development of rheumatic IRAE, thus there are no population estimates for prevalence and incidence of rheumatic IRAE.

As a result of inconsistent recognition and reporting of rheumatic IRAE, the epidemiology, clinical features, and optimal treatment are unknown. There is also a lack of recognition among the rheumatology community, as referrals have been inconsistent. Even if patients are referred to rheumatology, no evidence-based recommendations for evaluation and treatment tailored to patients who have active malignancy are available.

\section{A RAPIDLY EXPANDING AREA}

As we begin to understand the scope of rheumatic IRAE, it is important to note that ICIs are being used with increasing frequency by oncologists. Excitement surrounding this group of therapies will lead to exponential growth in use for several reasons.

\section{Expanding indications of existing drugs}

In the past 2 years, ICIs went from having a single indication, metastatic melanoma, to having five approved indications to date (melanoma, RCC, NSCLC, urothelial carcinoma and Hodgkin's lymphoma). There have been positive results reported in other tumours such as colorectal cancers with mismatch repair defects, ${ }^{20}$ Merkel Cell carcinoma, ${ }^{21}$ and in relapse after haematopoietic stem cell transplant for haematologic malignancies. ${ }^{22}$ The non-specific mechanism of action of these drugs along with these emerging data indicate that we should expect to see approvals of ICIs for many new indications in the upcoming years.

\section{New targets}

Presently, hundreds of trials are ongoing internationally using ICIs. Other immune checkpoint targets with drugs in clinical trials include B7, CD137, T-cell immunoglobulin and mucin domain-3 (TIM-3) and lymphocyte activation gene-3 (LAG-3). ${ }^{23}$ It remains to be seen how the risk of IRAE may differ by which immune checkpoint is targeted, but at minimum, approvals of drugs targeting these related pathways will increase the use of ICIs.

\section{Combination therapy}

There is currently one FDA-approved combination ICI regimen, ipilimumab and nivolumab for metastatic melanoma. The combination group in the phase III trial for that regimen had higher rates of IRAEs than both monotherapy groups. ${ }^{24}$ In our series of 13 patients, 8 were on combination therapy, while 5 were on monotherapy. ${ }^{13}$ Many of the ongoing trials use ICIs in combination. Thus, the rate of IRAE is likely to increase as more regimens are studied and indications approved.

\section{Earlier use of ICls}

Among the many trials of immunotherapy are those focusing on ICIs as neo-adjuvant therapy and in earlier stage disease. This is important for two reasons. First, perhaps more importantly, earlier use of ICIs means a higher likelihood of inducing a lasting remission and thus a longer lifespan in which ongoing management of rheumatic IRAEs may be required. Second, earlier use of the drugs and approval of ICIs as first-line therapy will expand the total number of patients exposed.

\section{Use of ICIs in those with pre-existing autoimmunity}

In clinical trials of immunotherapy, known autoimmune disease has been an exclusion criterion. Now that several ICIs are approved and can be used as standard of care therapy, they can potentially be given to patients with pre-existing autoimmune disease. In one study evaluating 30 patients with known autoimmune disease treated with ipilimumab, 8 experienced flares of their underlying autoimmune disease, and 10 developed a new IRAE. $^{25}$ These data suggest that as ICIs are given to patients with known autoimmunity, the rates of IRAEs, including rheumatic IRAEs, will be greater.

\section{WHY THE RHEUMATOLOGIST MATTERS}

Rheumatologists have a unique role to play in managing IRAE. Patients with rheumatic IRAE differ from patients with traditional rheumatic disease in several important ways. First, it appears that their IRAEs do not behave identically to the corresponding classic rheumatic disease. Observations to date show a lack of the traditional autoantibodies associated with RA and Sjogren's 
syndrome in most patients with ICI-induced inflammatory arthritis and sicca syndrome, respectively. ${ }^{13}$ Also, the steroid requirements of therapy for control may be much higher than typically required to manage 'classic' inflammatory arthritis. ${ }^{13}$ Importantly, all patients with rheumatic IRAE have or recently had advanced stage cancer, so there would be concerns about using immunosuppression in them. These concerns are compounded in patients whose cancer has responded to immunotherapy with further concerns about impairing a salutary response to the malignancy with concomitant or subsequent immunosuppression.

\section{Diagnosis and evaluation}

Rheumatologists can help in determining whether the symptoms experienced by patients on ICIs and those more commonly recognised by oncologists (eg, arthralgias, myalgias) represent a true inflammatory arthritis or other rheumatic IRAEs. Another area of importance will be determining whether patients presenting with symptoms may be manifesting exacerbation of a pre-existing autoimmune disease or really have a new ICI-emergent IRAE. Further, assessment by rheumatologists will be very important in recognising and describing the differences between ICI-induced rheumatic IRAEs and traditional rheumatic diseases to inform how evaluation and management should differ.

\section{Management of immunosuppression}

As rheumatologists, we are comfortable with adjusting biologics and conventional synthetic disease modifying anti-rheumatic drugs (DMARDs) to achieve optimal outcomes. Our familiarity with TNF inhibitors and other medications that may be needed to treat IRAEs put us in a position to monitor for toxicities and to consider combinatorial induction and/or tapering strategies using immunomodulators. The logistics of obtaining approval for biological medications in patients who may not meet classification criteria for traditional rheumatic diseases can also benefit from the rheumatologist's experience.

\section{CONCLUSIONS}

Rheumatic IRAEs are likely under-reported to date, and the trend towards vast expansion in use of ICI drugs suggests that ICI-induced rheumatic disease will be an increasing problem encountered by oncologists and rheumatologists in the near future and over time. Increased awareness of the potential for an array of rheumatic IRAEs is critical to early recognition, evaluation and therapy, which will hopefully foster better long-term outcomes. Careful tracking of patients treated with ICIs with a registry would provide more comprehensive epidemiologic data for rheumatic and other IRAEs. The expertise of rheumatologists will be critical to successful management of patients with ICI-induced rheumatic disease, particularly in the use of biologics and immunosuppressant/ immunomodulatory medications in general. Collaboration with oncology to research the pathogenesis of rheumatic IRAE and to define appropriate treatment algorithms will lead to better understanding of this growing phenomenon and identify potential targets for treatment.

Contributors LCC and COB planned the manuscript. All authors composed and edited the manuscript.

Funding This study was supported by Jerome L. Greene Foundation Scholar Award and National Institute of Arthritis and Musculoskeletal and Skin Diseases (P30-AR053503; K23-AR061439).

Competing interests COB has served as a consultant for Bristol-Myers Squibb.

Provenance and peer review Commissioned; externally peer reviewed.

Data sharing statement No additional data are available.

Open Access This is an Open Access article distributed in accordance with the Creative Commons Attribution Non Commercial (CC BY-NC 4.0) license, which permits others to distribute, remix, adapt, build upon this work noncommercially, and license their derivative works on different terms, provided the original work is properly cited and the use is non-commercial. See: http:// creativecommons.org/licenses/by-nc/4.0/

\section{REFERENCES}

1. Pardoll DM. The blockade of immune checkpoints in cancer immunotherapy. Nat Rev Cancer 2012;12:252-64.

2. Ansell SM, Lesokhin AM, Borrello I, et al. PD-1 blockade with nivolumab in relapsed or refractory Hodgkin's lymphoma. $N$ Engl J Med 2015;372:311-19.

3. Powles T, Eder JP, Fine GD, et al. MPDL3280A (anti-PD-L1) treatment leads to clinical activity in metastatic bladder cancer. Nature 2014;515:558-62.

4. Motzer RJ, Escudier B, McDermott DF, et al. Nivolumab versus everolimus in advanced renal-cell carcinoma. $N$ Engl $\mathrm{J}$ Med 2015;373:1803-13.

5. Brahmer J, Reckamp KL, Baas P, et al. Nivolumab versus docetaxel in advanced squamous-cell non-small-cell lung cancer. $N$ Engl J Med 2015;373:123-35.

6. Postow MA, Chesney J, Pavlick AC, et al. Nivolumab and ipilimumab versus ipilimumab in untreated melanoma. $N$ Engl $\mathrm{J}$ Med 2015;372:2006-17.

7. Robert C, Schachter J, Long GV, et al. Pembrolizumab versus ipilimumab in advanced melanoma. $N$ Engl J Med 2015;372:2521-32.

8. Weber JS, Kähler KC, Hauschild A. Management of immune-related adverse events and kinetics of response with ipilimumab. J Clin Oncol 2012;30:2691-7.

9. Lord JD, Hackman RC, Moklebust A, et al. Refractory colitis following anti-CTLA4 antibody therapy: analysis of mucosal FOXP3+ T cells. Dig Dis Sci 2010;55:1396-405.

10. Tarhini AA, Zahoor $\mathrm{H}$, Lin $\mathrm{Y}$, et al. Baseline circulating IL-17 predicts toxicity while TGF-beta1 and IL-10 are prognostic of relapse in ipilimumab neoadjuvant therapy of melanoma. $J$ Immunother Cancer 2015;3:39.

11. Law-Ping-Man S, Martin A, Briens E, et al. Psoriasis and psoriatic arthritis induced by nivolumab in a patient with advanced lung cancer. Rheumatology (Oxford) 2016.

12. Chan MM, Kefford RF, Carlino M, et al. Arthritis and tenosynovitis associated with the anti-PD1 antibody pembrolizumab in metastatic melanoma. J Immunother 2015;38:37-9.

13. Cappelli LC, Gutierrez AK, Baer AN, et al. Inflammatory arthritis and sicca syndrome induced by nivolumab and ipilimumab. Ann Rheum Dis 2016.

14. Manusow JS, Khoja L, Pesin N, et al. Retinal vasculitis and ocular vitreous metastasis following complete response to PD-1 inhibition in a patient with metastatic cutaneous melanoma. $J$ Immunother Cancer 2014;2:41.

15. Minor DR, Bunker SR, Doyle J. Lymphocytic vasculitis of the uterus in a patient with melanoma receiving ipilimumab. $J$ Clin Oncol 2013;31:e356.

16. Sheik Ali S, Goddard AL, Luke JJ, et al. Drug-associated dermatomyositis following ipilimumab therapy: a novel immune-mediated adverse event associated with cytotoxic T-lymphocyte antigen 4 blockade. JAMA Dermatol 2015;151:195-9. 
17. Yoshioka M, Kambe N, Yamamoto Y, et al. Case of respiratory discomfort due to myositis after administration of nivolumab. $J$ Dermatol 2015;42:1008-9.

18. Khoja L, Maurice $\mathrm{C}$, Chappell M, et al. Eosinophilic fasciitis and acute encephalopathy toxicity from pembrolizumab treatment of a patient with metastatic melanoma. Cancer Immunol Res 2016;4:175-8.

19. Fadel F, El Karoui K, Knebelmann B. Anti-CTLA4 antibody-induced lupus nephritis. N Engl J Med 2009;361:211-12.

20. Le DT, Uram JN, Wang H, et al. PD-1 blockade in tumors with mismatch-repair deficiency. N Engl J Med 2015;372:2509-20.

21. Nghiem PT, Bhatia S, Lipson EJ, et al. PD-1 blockade with pembrolizumab in advanced Merkel-cell carcinoma. N Engl J Med 2016;374:2542-52.
22. Davids MS, Kim HT, Bachireddy $P$, et al. Ipilimumab for patients with relapse after allogeneic transplantation. $N$ Engl J Med 2016;375:143-53.

23. Topalian SL, Drake CG, Pardoll DM. Immune checkpoint blockade: a common denominator approach to cancer therapy. Cancer Cell 2015;27:450-61.

24. Larkin J, Chiarion-Sileni V, Gonzalez R, et al. Combined nivolumab and ipilimumab or monotherapy in untreated melanoma. $N$ Engl J Med 2015;373:23-34.

25. Johnson DB, Sullivan RJ, Ott PA, et al. Ipilimumab therapy in patients with advanced melanoma and preexisting autoimmune disorders. JAMA Oncol 2016;2 234-40. 